\title{
On General Sum Approximations of Irrational Numbers
}

\author{
Ivan Georgiev ${ }^{1}$ \\ Lars Kristiansen ${ }^{2,3}$ \\ Frank Stephan ${ }^{4}$ \\ 1 Department of Mathematics and Physics, Faculty of Natural Sciences, \\ University "Prof. d-r Asen Zlatarov", Burgas 8010, Bulgaria* \\ ivandg@yahoo.com \\ 2 Department of Mathematics, University of Oslo, Norway \\ 3 Department of Informatics, University of Oslo, Norway \\ larsk@math.uio.no \\ 4 Department of Mathematics and School of Computing, \\ National University of Singapore, Singapore 119076, Republic of Singapore ${ }^{\star \star}$ \\ fstephan@comp.nus.edu.sg
}

\section{Introduction and Basic Definitions}

There are numerous ways to represent real numbers. We may use, e.g., Cauchy sequences, Dedekind cuts, numerical base-10 expansions, numerical base-2 expansions and continued fractions. If we work with full Turing computability, all these representations yield the same class of real numbers. If we work with some restricted notion of computability, e.g., polynomial time computability or primitive recursive computability, they do not. This phenomenon has been investigated over the last seven decades by Specker [13], Mostowski [8], Lehman [10], Ko [3, 4], Labhalla \& Lombardi [9], Georgiev [1], Kristiansen [5, 6] and quite a few more. Georgiev et al. [2] is an extended version of the current paper.

Irrational numbers can be represented by infinite sums. Sum approximations from below and above were introduced in Kristiansen [5] and studied further in Kristiansen [6]. Every irrational number $\alpha$ between 0 and 1 can be uniquely written as an infinite sum of the form

$$
\alpha=0+\frac{D_{1}}{b^{k_{1}}}+\frac{D_{2}}{b^{k_{2}}}+\frac{D_{3}}{b^{k_{3}}}+\ldots
$$

where

$-b \in \mathbb{N} \backslash\{0,1\}$ and $\mathrm{D}_{i} \in\{1, \ldots, b-1\}$ (note that $\mathrm{D}_{i} \neq 0$ for all $i$ )

* I. Georgiev has been supported by the Bulgarian National Science Fund through the project "Models of computability", DN-02-16/19.12.2016.

${ }^{\star \star}$ F. Stephan has been supported in part by the Singapore Ministry of Education Academic Research Fund grant MOE2016-T2-1-019 / R146-000-234-112. 
$-k_{i} \in \mathbb{N} \backslash\{0\}$ and $k_{i}<k_{i+1}$.

Let $\hat{A}_{b}^{\alpha}(i)=\mathrm{D}_{i} b^{-k_{i}}$ for $i>0$ (and let $\hat{A}_{b}^{\alpha}(0)=0$ ). The rational number $\sum_{i=1}^{n} \hat{A}_{b}^{\alpha}(i)$ is an approximation of $\alpha$ that lies below $\alpha$, and we will say that the function $\hat{A}_{b}^{\alpha}$ is the base-b sum approximation from below of $\alpha$. The base- $b$ sum approximation from above of $\alpha$ is a symmetric function $\breve{A}_{b}^{\alpha}$ such that $1-$ $\sum_{i=1}^{n} \check{A}_{b}^{\alpha}(i)$ is an approximation of $\alpha$ that lies above $\alpha$ (and we have $\sum_{i=1}^{\infty} \hat{A}_{b}^{\alpha}(i)+$ $\left.\sum_{i=1}^{\infty} \check{A}_{b}^{\alpha}(i)=1\right)$. Let $\mathcal{S}$ be any class of subrecursive functions which is closed under primitive recursive operations. Furthermore, let $\mathcal{S}_{b \uparrow}$ denote the set of irrational numbers that have a base- $b$ sum approximation from below in $\mathcal{S}$, and let $\mathcal{S}_{b \downarrow}$ denote the set of irrational numbers that have a base- $b$ sum approximation from above in $\mathcal{S}$. It is proved in [6] that $\mathcal{S}_{b \uparrow}$ and $\mathcal{S}_{b \downarrow}$ are incomparable classes, that is, $\mathcal{S}_{b \uparrow} \nsubseteq \mathcal{S}_{b \downarrow}$ and $\mathcal{S}_{b \downarrow} \nsubseteq \mathcal{S}_{b \uparrow}$. Another interesting result proved in [6] is that $\mathcal{S}_{a \downarrow} \subseteq \mathcal{S}_{b \downarrow}$ iff $\mathcal{S}_{a \uparrow} \subseteq \mathcal{S}_{b \uparrow}$ iff every prime factor of $b$ is a prime factor of $a$.

In this paper we prove some results on general sum approximations. The general sum approximation from below of $\alpha$ is the function $\hat{G}^{\alpha}: \mathbb{N} \times \mathbb{N} \rightarrow \mathbb{Q}$ defined by $\hat{G}^{\alpha}(b, n)=\hat{A}_{b}^{\alpha}(n)$; let $\hat{G}^{\alpha}(b, n)=0$ if $b<2$. The general sum approximation from above of $\alpha$ is the function $\breve{G}^{\alpha}: \mathbb{N} \times \mathbb{N} \rightarrow \mathbb{Q}$ defined by $\check{G}^{\alpha}(b, n)=\check{A}_{b}^{\alpha}(n)$; let $\check{G}^{\alpha}(b, n)=0$ if $b<2$. Let $\mathcal{S}$ be any class of subrecursive functions which is closed under primitive recursive operations. Furthermore, let $\mathcal{S}_{g \uparrow}$ denote the set of irrational numbers that have a general sum approximation from below in $\mathcal{S}$, and let $\mathcal{S}_{g \downarrow}$ denote the set of irrational numbers that have a general sum approximation from above in $\mathcal{S}$.

It was proved in [5] that $\mathcal{S}_{g \uparrow} \cap \mathcal{S}_{g \downarrow}$ contains exactly the irrational numbers that have a continued fraction in the class $\mathcal{S}$. In this paper we prove that $\mathcal{S}_{g \uparrow} \neq \mathcal{S}_{g \downarrow}$. Moreover, we prove that

$$
\mathcal{S}_{g \downarrow} \neq \bigcap_{b=2}^{\infty} \mathcal{S}_{b \downarrow} \quad \text { and } \quad \mathcal{S}_{g \uparrow} \neq \bigcap_{b=2}^{\infty} \mathcal{S}_{b \uparrow} .
$$

Some might find it interesting (at least the authors do) that we manage to complete all our proof without resorting to the standard computability-theoretic machinery involving enumerations, universal functions, diagonalizations, and so on. We prove our results by providing natural irrationals numbers (the numbers are natural in the sense that they have neat and transparent definitions).

\section{Preliminaries}

We will restrict our attention to real numbers between 0 and 1 .

A base is a natural number strictly greater than 1 , and a base-b digit is a natural number in the set $\{0,1, \ldots, b-1\}$.

Let $b$ be a base, and let $\mathrm{D}_{1}, \ldots, \mathrm{D}_{n}$ be base- $b$ digits. We will use $\left(0 . \mathrm{D}_{1} \mathrm{D}_{2} \ldots \mathrm{D}_{n}\right)_{b}$ to denote the rational number $\sum_{i=1}^{n} \mathrm{D}_{i} b^{-i}$. 
Let $D_{1}, D_{2}, \ldots$ be an infinite sequence of base- $b$ digits. We say that $\left(0 . D_{1} D_{2} \ldots\right)_{b}$ is the base-b expansion of the real number $\alpha$ if for all $n \geq 1$ we have

$$
\left(0 . \mathrm{D}_{1} \mathrm{D}_{2} \ldots \mathrm{D}_{n}\right)_{b} \leq \alpha<\left(0 . \mathrm{D}_{1} \mathrm{D}_{2} \ldots \mathrm{D}_{n}\right)_{b}+b^{-n} .
$$

Every real number $\alpha$ has a unique base- $b$ expansion (note the strict inequality). When $\alpha=\left(0 . \mathrm{D}_{1} \mathrm{D}_{2} \ldots \mathrm{D}_{n}\right)_{b}$ for some $n$ with $\mathrm{D}_{n} \neq 0$, we say that $\alpha$ has a finite base-b expansion of length $n$. Otherwise, we say that $\alpha$ has an infinite base- $b$ expansion, and this infinite base- $b$ expansion is periodic iff $\alpha$ is rational. More concretely, if $\alpha=c d^{-1}$ for non-zero relatively prime $c, d \in \mathbb{N}$, then the base- $b$ expansion of $\alpha$ is of the form $0 . \mathrm{D}_{1} \ldots \mathrm{D}_{s}\left(\mathrm{D}_{s+1} \ldots \mathrm{D}_{t}\right)^{\omega}$ which we use as shorthand for the infinite sequence $0 . \mathrm{D}_{1} \ldots \mathrm{D}_{s} \mathrm{D}_{s+1} \ldots \mathrm{D}_{t} \mathrm{D}_{s+1} \ldots \mathrm{D}_{t} \mathrm{D}_{s+1} \ldots \mathrm{D}_{t} \ldots$ The number $s$ is the largest natural number such that $p^{s}$ divides $d$ for some prime factor $p$ of $b$. The length of the period $t-s$ is the multiplicative order of $b$ modulo $d_{1}$ where $d_{1}$ is the largest divisor of $d$ relatively prime with $b$. It follows straightforwardly that $t<d$. Of course, $\alpha$ has a finite base- $b$ expansion iff $d_{1}=1$, that is, iff every prime factor of $d$ is a prime factor of $b$.

We assume the reader is familiar with subrecursion theory and subrecursive functions. An introduction to the subject can be found in [11] or [12].

A function $\phi$ is elementary in a function $\psi$, written $\phi \leq_{E} \psi$, if $\phi$ can be generated from the initial functions $\psi, 2^{x}$, max, $0, S$ (successor), $I_{i}^{n}$ (projections) by composition and bounded primitive recursion. A function $\phi$ is elementary if $\phi \leq_{E} 0$. A function $\phi$ is primitive recursive in a function $\psi$, written $\phi \leq_{P R} \psi$, if $\phi$ can be generated from the initial functions by composition and (unbounded) primitive recursion. A function $\phi$ is primitive recursive if $\phi \leq_{P R} 0$.

Subrecursive functions in general, and elementary functions in particular, are formally functions over natural numbers $(\mathbb{N})$. We assume some coding of integers $(\mathbb{Z})$ and rational numbers $(\mathbb{Q})$ into the natural numbers. We consider such a coding to be trivial. Therefore we allow for subrecursive functions from rational numbers into natural numbers, from pairs of rational numbers into rational numbers, etc., with no further comment. Uniform systems for coding finite sequences of natural numbers are available inside the class of elementary functions. Hence, for any reasonable coding, basic operations on rational numbers - like e.g. addition, subtraction and multiplication - will obviously be elementary. It is also obvious that there is an elementary function $\psi(q, i, b)$ that yields the $i^{\text {th }}$ digit in the base- $b$ expansion of the rational number $q$.

A function $f: \mathbb{N} \rightarrow \mathbb{N}$ is honest if it is monotonically increasing $(f(x) \leq f(x+1))$, dominates $2^{x}\left(f(x) \geq 2^{x}\right)$ and has elementary graph (the relation $f(x)=y$ is elementary).

A class of functions $\mathcal{S}$ is subrecursive class if $\mathcal{S}$ is an efficiently enumerable class of computable total functions. For any subrecursive class $\mathcal{S}$ there exists an honest function $f$ such that $f \notin \mathcal{S}$ (see Section 8 of [5] for more details).

More on elementary functions, primitive recursive functions and honest functions can be found in Section 2 of [5] and in [7]. 


\section{Irrational Numbers with Interesting Properties}

Definition 1. Let $P_{i}$ denote the $i^{\text {th }}$ prime $\left(P_{0}=2, P_{1}=3, \ldots\right)$. We define the auxiliary function $g$ by

$$
g(0)=1 \quad \text { and } \quad g(j+1)=P_{j}^{2(j+2)(g(j)+1)^{3}} .
$$

For any honest function $f$ and any $n \in \mathbb{N}$, we define the rational number $\alpha_{n}^{f}$ and the irrational number $\alpha^{f}$ by

$$
\alpha_{n}^{f}=\sum_{i=0}^{n} P_{i}^{-h(i)} \quad \text { and } \quad \alpha^{f}=\lim _{n \rightarrow \infty} \alpha_{n}^{f}
$$

where $h(i)=g(f(i)+i)($ for any $i \in \mathbb{N})$.

It is easy to see that both $g$ and $h$ are strictly increasing honest functions. Moreover, we have

$$
P_{n}^{2(n+2)(h(n)+1)^{3}}<h(n+1)
$$

for any $n \in \mathbb{N}$. Thus the function $h$ possesses a growth property which $f$ might not possess. We will need this property. This explains why we introduce the function $g$ in the definition of $\alpha^{f}$.

When $f$ is a fixed honest function, we abbreviate $\alpha_{j}^{f}$ and $\alpha^{f}$ to $\alpha_{j}$ and $\alpha$, respectively.

The next lemma is easily proven using the preliminaries on base- $b$ expansions.

Lemma 2. For any $j \in \mathbb{N}$ and any base b, we have

(i) if $P_{i}$ divides $b$ for all $i \leq j$, then $\alpha_{j}$ has a finite base- $b$ expansion of length $h(j)$

(ii) if $P_{i}$ does not divide $b$ for some $i \leq j$, then $\alpha_{j}$ has an infinite (periodic) base-b expansion.

Lemma 3. Let

- $b$ be any base, and let $j \in \mathbb{N}$ be such that $P_{j}>b$

- $\left(0 . D_{1} D_{2} \ldots\right)_{b}$ be the base-b expansion of $\alpha_{j}$

- $\left(0 . \dot{D}_{1} \dot{D}_{2} \ldots\right)_{b}$ be the base-b expansion of $\alpha_{j+1}$

- $M=M(j)=P_{j}^{(j+1) h(j)}$ and $M^{\prime}=M^{\prime}(j)=h(j+1)$.

Then

(i) there are no more than $M$ consecutive zeros in the base-b expansion of $\alpha_{j}$, that is, for any $k \in \mathbb{N} \backslash\{0\}$ there exists $i \in \mathbb{N}$ such that

$$
k \leq i<k+M \quad \text { and } \quad D_{i} \neq 0
$$


(ii) the first $M^{\prime}-M$ digits of the base-b expansions of $\alpha_{j}$ and $\alpha_{j+1}$ coincide, that is

$$
i \leq M^{\prime}-M \Rightarrow D_{i}=\dot{D}_{i}
$$

and moreover, these digits also coincide with the corresponding digits of the base-b expansion of $\alpha$.

Proof. By Lemma 2 (ii), $\alpha_{j}$ has an infinite periodic base- $b$ expansion of the form $0 . \mathrm{D}_{1} \ldots \mathrm{D}_{s}\left(\mathrm{D}_{s+1} \ldots \mathrm{D}_{t}\right)^{\omega}$ with $s<t$. Using the preliminaries on base- $b$ expansions we obtain

$$
t-s \leq t<\prod_{i=0}^{j} P_{i}^{h(i)} \leq P_{j}^{(j+1) h(j)}=M .
$$

Thus (i) holds since every $M$ consecutive digits of $\alpha_{j}$ contain all the digits $\mathrm{D}_{s+1}, \ldots, \mathrm{D}_{t}$ of at least one period.

We turn to the proof of (ii). We have

$$
\alpha_{j}<\alpha_{j+1}=\alpha_{j}+P_{j+1}^{-h(j+1)} \leq \alpha_{j}+b^{-M^{\prime}}
$$

since $b^{M^{\prime}}<P_{j}^{M^{\prime}}=P_{j}^{h(j+1)}<P_{j+1}^{h(j+1)}$. At least one digit in the period $\mathrm{D}_{s+1} \ldots \mathrm{D}_{t}$ is different from $b-1$, and the length of the period is $t-s$. Thus (3) entails

$$
\mathrm{D}_{i}=\dot{\mathrm{D}}_{i} \text { for any } i \leq M^{\prime}-(t-s) \text {. }
$$

It follows from (2) and (4) that the first $M^{\prime}-M$ digits of the base- $b$ expansions of $\alpha_{j}$ and $\alpha_{j+1}$ coincide. Moreover, since $M^{\prime}(j)$ is strictly increasing in $j$,

$$
\alpha_{j}<\alpha_{j+k} \leq \alpha_{j}+\sum_{i<k} b^{-M^{\prime}(j+i)} \leq \alpha_{j}+b^{-M^{\prime}(j)+1}
$$

for any $k \geq 1$. Letting $k \rightarrow \infty$ we obtain as above that the first $M^{\prime}-M$ digits of $\alpha_{j}$ and $\alpha$ coincide.

Theorem 4. Let $f$ be any honest function, and let $b$ be any base. The function $\hat{A}_{b}^{\alpha^{f}}$ is elementary.

Proof. Fix the least $m$ such that $P_{m}>b$. We will use the functions $M$ and $M^{\prime}$ from Lemma 3 . We will argue that we can compute the rational number $\hat{A}_{b}^{\alpha}(n)$ elementarily in $n$ when $n \geq M(m)$. Note that $M(m)$ is a fixed number (it does not depend on $n)$. Thus, we can compute $\hat{A}_{b}^{\alpha}(n)$ by a trivial algorithm when $n<M(m)$ (use a huge table).

Assume $n \geq M(m)$. We will now give an algorithm for computing $\hat{A}_{b}^{\alpha}(n)$ elementarily in $n$.

Step 1 of the algorithm: Compute (the unique) $j$ such that

$$
M(j) \leq n<M(j+1)
$$


(end of Step 1).

Step 1 is a computation elementary in $n$ since $M$ has elementary graph. So is Step 2 as $M^{\prime}$ also has elementary graph.

Step 2 of the algorithm: Check if the following relation holds:

$$
n^{2}+1<M^{\prime}(j)-M(j) .
$$

If it holds, carry out Step 3A, otherwise, carry out Step 3B (end of Step 2).

Step $3 A$ of the algorithm: Compute $\alpha_{j}$. Then compute base- $b$ digits $\mathrm{D}_{1}, \ldots, \mathrm{D}_{n^{2}+1}$ such that

$$
\left(0 . \mathrm{D}_{1} \mathrm{D}_{2} \ldots \mathrm{D}_{n^{2}+1}\right)_{b} \leq \alpha_{j}<\left(0 . \mathrm{D}_{1} \mathrm{D}_{2} \ldots \mathrm{D}_{n^{2}+1}\right)_{b}+b^{-\left(n^{2}+1\right)} .
$$

Find $k$ such that $\mathrm{D}_{k}$ is the $n^{\text {th }}$ digit different from 0 in the sequence $\mathrm{D}_{1}, \ldots, \mathrm{D}_{n^{2}+1}$. Give the output $\mathrm{D}_{k} b^{-k}$ (end of Step 3A).

Recall that $\alpha_{j}=\sum_{i=0}^{j} P_{i}^{-h(i)}$. We can compute $\alpha_{j}$ elementarily in $n$ since $h(0), h(1), \ldots, h(j)<M(j) \leq n$ and $h$ is honest. Thus, we can also compute the base- $b$ digits $\mathrm{D}_{1}, \mathrm{D}_{2}, \ldots, \mathrm{D}_{n^{2}+1}$ elementarily in $n$. In order to prove that our algorithm is correct, we must verify that

(A) at least $n$ of the digits $\mathrm{D}_{1}, \mathrm{D}_{2}, \ldots, \mathrm{D}_{n^{2}+1}$ are different from 0 , and

(B) $\mathrm{D}_{1}, \mathrm{D}_{2}, \ldots, \mathrm{D}_{n^{2}+1}$ coincide with the first $n^{2}+1$ digits of $\alpha$.

By Lemma 3 (i) the sequence $\mathrm{D}_{k M(j)+1}, \mathrm{D}_{k M(j)+2}, \ldots, \mathrm{D}_{(k+1) M(j)}$ contains at least one non-zero digit (for any $k \in \mathbb{N}$ ). Thus, (A) holds since $n \geq M(j)$. Using (6) and Lemma 3 (ii) we see that (B) also holds. This proves that the output $\mathrm{D}_{k} b^{-k}=\hat{A}_{b}^{\alpha}(n)$.

Step $3 B$ of the algorithm: Compute $\alpha_{j+1}$ and $M(j+1)$. Then proceed as in Step $3 \mathrm{~A}$ with $\alpha_{j+1}$ in place of $\alpha_{j}$ and $n M(j+1)$ in place of $n^{2}$ (end of Step 3B).

Step 3B is only executed when $M^{\prime}(j)-M(j) \leq n^{2}+1$. Thus, we have $M^{\prime}(j)=$ $h(j+1) \leq n^{2}+n+1$. This entails that we can compute $h(j+1)-$ and also $\alpha_{j+1}$ and $M(j+1)$ - elementarily in $n$. It follows easily from (1) that

$$
M(j+1)^{2}+M(j+1)+1<M^{\prime}(j+1)
$$

which together with (5) imply

$$
n M(j+1)+1<M^{\prime}(j+1)-M(j+1) .
$$

As in Step 3A, there will be at least $n$ non-zero digits among the first $n M(j+1)$ digits of $\alpha_{j+1}$. Moreover, the first $n M(j+1)$ digits of $\alpha_{j+1}$ coincide with the corresponding digits of $\alpha$.

Theorem 5. Let $f$ be any honest function. We have $f \leq_{P R} \hat{G}^{\alpha^{f}}$ ( $f$ is primitive recursive in $\hat{G}^{\alpha^{f}}$ ). 
Proof. Fix $n \in \mathbb{N}$, and let $b$ be the base $b=\prod_{i=0}^{n} P_{i}$. By Lemma (2) (i), $\alpha_{n}$ has a finite base- $b$ expansion of length $h(n)$. By the definition of $\alpha$, we have

$$
\alpha=\alpha_{n}+P_{n+1}^{-h(n+1)}+P_{n+2}^{-h(n+2)}+\ldots .
$$

It follows that for any $j>h(n)$

$$
\hat{G}^{\alpha}(b, j) \leq P_{n+1}^{-h(n+1)}+P_{n+2}^{-h(n+2)}+\ldots,
$$

which easily implies $\hat{G}^{\alpha}(b, j) \leq P_{n+1}^{-h(n+1)+1}$ (use that $h$ is strictly increasing). Hence we also have $\left(\hat{G}^{\alpha}(b, j)\right)^{-1} \geq P_{n+1}^{h(n+1)-1}>h(n+1)-1$ for any $j>h(n)$.

The considerations above show that we can compute $h(n+1)$ by the following algorithm:

- assume that $h(n)$ is computed;

- compute $b=\prod_{i=0}^{n} P_{i}$

- search for $y$ such that $y<\left(\hat{G}^{\alpha}(b, h(n)+1)\right)^{-1}+1$ and $h(n+1)=y$;

- give the output $y$.

This algorithm is primitive recursive in $\hat{G}^{\alpha}$ : The computation of $b$ is an elementary computation. The relation $h(x)=y$ is elementary, and thus the search for $y$ is elementary in $h(n)$ and $\hat{G}^{\alpha}$. This proves that $h$ is primitive recursive in $\hat{G}^{\alpha}$. But then $f$ will also be primitive recursive in $\hat{G}^{\alpha}$ as the graph of $f$ is elementary and $f(n) \leq h(n)$ (for any $n \in \mathbb{N}$ ). This proves that $f \leq_{P R} \hat{G}^{\alpha}$.

Theorem 6. Let $f$ be any honest function. There exists an elementary function $\check{T}: \mathbb{Q} \rightarrow \mathbb{Q}$ such that (i) $\check{T}(q)=0$ if $q<\alpha^{f}$ and (ii) $q>\check{T}(q)>\alpha^{f}$ if $q>\alpha^{f}$.

Proof. In addition to the sequence $\alpha_{j}$ we need the sequence $\beta_{j}$ given by

$$
\beta_{0}=P_{0}^{-h(0)+1}=2^{-h(0)+1} \text { and } \beta_{j+1}=\alpha_{j}+P_{j+1}^{-h(j+1)+1} .
$$

Observe that we have $\alpha<\beta_{j}$ for all $j \in \mathbb{N}$.

Now we will explain an algorithm that computes a function $\check{T}$ with the properties (i) and (ii).

Step 1 of the algorithm: The input is the rational number $q$. We can w.l.o.g. assume that $0<q<1$. Pick any $m^{\prime}, n \in \mathbb{N}$ such that $q=m^{\prime} n^{-1}$ and $n \geq h(0)$. Find $m \in \mathbb{N}$ such that $q=m\left(P_{0} P_{1} \ldots P_{n}\right)^{-n}$, and compute the base $b$ such that $b=\prod_{i=0}^{n} P_{i}$ (end of Step 1).

The rational number $q$ has a finite base- $b$ expansion of length $s$ where $s \leq n$. Moreover, the rational numbers $\alpha_{0}, \alpha_{1}, \ldots, \alpha_{n}$ and $\beta_{0}, \beta_{1}, \ldots, \beta_{n}$ also have finite base- $b$ expansions.

Step 2 of the algorithm: Compute (the unique) natural number $j<n$ such that

$$
h(j) \leq n<h(j+1) .
$$


Furthermore, compute $\alpha_{0}, \alpha_{1}, \ldots, \alpha_{j}$ and $\beta_{0}, \beta_{1}, \ldots, \beta_{j}$ (end of Step 2).

All the numbers $h(0), h(1), \ldots h(j)$ are less than or equal to $n$, and $h$ has elementary graph. This entails that Step 2 is elementary in $n$ (and also in $q$ ).

Step 3 of the algorithm: If $q \leq \alpha_{k}$ for some $k \leq j$, give the output 0 and terminate. If $\beta_{k}<q$ for some $k \leq j$, give the output $\beta_{k}$ and terminate (end of Step 3).

Step 3 is obviously elementary in $q$ and gives the correct output.

If the algorithm has not yet terminated, we have $\alpha_{j}<q \leq \beta_{j}$. Now

$$
q \leq \beta_{j+1} \Leftrightarrow q-\alpha_{j} \leq P_{j+1}^{-h(j+1)+1} \Leftrightarrow P_{j+1}^{h(j+1)} \leq\left(q-\alpha_{j}\right)^{-1} P_{j+1} .
$$

We have determined $\alpha_{j}$, and $h$ is an honest function. This makes it possible to check elementarily if $q \leq \beta_{j+1}$ : Search for $y<\left(q-\alpha_{j}\right)^{-1} P_{j+1}$ such that $h(j+1)=y$. If no such $y$ exists, we have $q>\beta_{j+1}$. If such an $y$ exists, we have $q \leq \beta_{j+1}$ iff $P_{j+1}^{y} \leq\left(q-\alpha_{j}\right)^{-1} P_{j+1}$.

Step 4 of the algorithm: Search for $y<\left(q-\alpha_{j}\right)^{-1} P_{j+1}$ such that $y=h(j+1)$. If the search is successful and $P_{j+1}^{y} \leq\left(q-\alpha_{j}\right)^{-1} P_{j+1}$, go to Step 5 , otherwise go to Step 6B (end of Step 4).

Clearly, Step 4 is elementary in $q$. If $q \leq \beta_{j+1}$, the next step is Step 5 (and we have computed $y=h(j+1)$ ). If $\beta_{j+1}<q$, the next step is Step 6B.

Step 5 of the algorithm: Compute $\alpha_{j+1}$ and $\beta_{j+1}$. If $q \leq \alpha_{j+1}$, give the output 0 and terminate. If $\alpha_{j+1}<q$, search for $z<\left(q-\alpha_{j+1}\right)^{-1} P_{j+2}$ such that $z=$ $h(j+2)$. If the search is successful and $P_{j+2}^{z} \leq\left(q-\alpha_{j+1}\right)^{-1} P_{j+2}$, give the output 0 and terminate, otherwise, go to Step 6A (end of Step 5).

Step 5 is elementary in $q$ since we have computed $h(j+1)$ in Step 4 . If the algorithm terminates because $q \leq \alpha_{j+1}$, we obviously have $q<\alpha$ and the output is correct. If $q>\alpha_{j+1}$, the algorithm will not proceed to Step 6A iff $\alpha_{j+1}<q \leq$ $\beta_{j+2}$. So assume that $\alpha_{j+1}<q \leq \beta_{j+2}$. It is not hard to show that the first $h(j+1)$ digits of $\alpha_{j+1}, \alpha, \beta_{j+2}$ coincide, and moreover, $h(j+1)>n \geq s$ (recall that $s$ is the length of the base- $b$ expansion of $q$ ). Thus, we have $q<\alpha$, and the algorithm gives the correct output, namely 0 . If the algorithm proceeds with Step $6 \mathrm{~A}$, we have $\beta_{j+2}<q$.

Step 6A of the algorithm: Compute the least $t$ such that $b^{t}>\left(q-\alpha_{j+1}\right)^{-1}$. Search for $u<\left(q-b^{-t}-\alpha_{j+1}\right)^{-1} P_{j+2}$ such that $u=h(j+2)$. If the search is successful and $P_{j+2}^{u}<\left(q-b^{-t}-\alpha_{j+1}\right)^{-1} P_{j+2}$, give the output $\beta_{j+2}$ and terminate, otherwise, give the output $q-b^{-t}$ and terminate (end of Step 6A).

It is easy to see that Step $6 \mathrm{~A}$ is elementary in $q$ : we can compute $t$ elementarily in $q, b$ and $\alpha_{j+1}$ (and we have already computed $b$ and $\alpha_{j+1}$ elementarily in $q$ ). When the execution of the step starts, we have $\beta_{j+2}<q$ (thus, $\beta_{j+2}$ will be a correct output, but we do not yet know if we will be able to compute $\beta_{j+2}$ ). If the search for $u$ is successful, we have $u=h(j+2)$. Then, we can compute $\beta_{j+2}$ elementarily in $u$, and give $\beta_{j+2}$ as output. We also know that the search for $u$ 
is successful iff $q-b^{-t}<\beta_{j+2}$. Thus, if the search for $u$ is not successful, we have $\alpha<\beta_{j+2} \leq q-b^{-t}<q$, and we can give the correct output $q-b^{-t}$.

Step $6 B$ of the algorithm: Exactly the same as $6 \mathrm{~A}$, but replace $j+1$ and $j+2$ by $j$ and $j+1$, respectively (end of Step $6 B$ ).

The argument for correctness of Step $6 \mathrm{~B}$ is the same as for Step $6 \mathrm{~A}$, just replace $j+1$ and $j+2$ by $j$ and $j+1$, respectively, and note that we have $\beta_{j+1}<q$ when the execution of the step starts.

Definition 7. A function $D: \mathbb{Q} \rightarrow\{0,1\}$ is a Dedekind cut of the real number $\beta$ when $D(q)=0$ iff $q<\beta$.

Corollary 8. Let $f$ be any honest function. The Dedekind cut of the real number $\alpha^{f}$ is elementary.

Proof. By Theorem 6 there is an elementary function $\check{T}$ such that $\check{T}(q)=0$ iff $q<\alpha^{f}$. Let $D(q)=0$ if $\check{T}(q)=0$, and let $D(q)=1$ if $\check{T}(q) \neq 0$. The function $D$ is elementary since $\check{T}$ is elementary. Moreover, $D$ is the Dedekind cut of $\alpha^{f}$.

\section{Main Results}

Theorem 9. For any subrecursive class $\mathcal{S}$ that is closed under primitive recursive operations, we have

$$
\text { (i) } \mathcal{S}_{g \downarrow} \subset \bigcap_{b} \mathcal{S}_{b \downarrow} \quad \text { and } \quad(\text { ii }) \mathcal{S}_{g \uparrow} \subset \bigcap_{b} \mathcal{S}_{b \uparrow} .
$$

Proof. The inclusion $\mathcal{S}_{g \uparrow} \subseteq \bigcap_{b} \mathcal{S}_{b \uparrow}$ is trivial. Pick an honest function $f$ such that $f \notin \mathcal{S}$. By Theorem 4, we have $\alpha^{f} \in \bigcap_{b} \mathcal{S}_{b \uparrow}$. By Theorem 5, we have $\alpha^{f} \notin \mathcal{S}_{g \uparrow}$. This proves that $\mathcal{S}_{g \uparrow} \subset \bigcap_{b} \mathcal{S}_{b \uparrow}$. The proof of (i) is symmetric.

Definition 10. A function $\hat{T}: \mathbb{Q} \rightarrow \mathbb{Q}$ is a trace function from below for the irrational number $\alpha$ when we have $q<\hat{T}(q)<\alpha$ for any $q<\alpha$. A function $\check{T}: \mathbb{Q} \rightarrow \mathbb{Q}$ is a trace function from above for the irrational number $\alpha$ when we have $\alpha<\check{T}(q)<q$ for any $q>\alpha$. A function $T: \mathbb{Q} \rightarrow \mathbb{Q}$ is a trace function for the irrational number $\alpha$ when we have $|\alpha-q|>|\alpha-T(q)|$ for any $q$.

For any subrecursive class $\mathcal{S}$, let $\mathcal{S}_{D}$ denote the set of irrational numbers that have a Dedekind cut in $\mathcal{S}$; let $\mathcal{S}_{T \uparrow}$ denote the set of irrational numbers that have a trace function from below in $\mathcal{S}$; let $\mathcal{S}_{T \downarrow}$ denote the set of irrational numbers that have a trace function from above in $\mathcal{S}$; let $\mathcal{S}_{T}$ denote the set of irrational numbers that have a trace function in $\mathcal{S}$; let $\mathcal{S}_{[]}$denote the set of irrational numbers that have a continued fraction in $\mathcal{S}$.

It is proved in [5] that we have $\mathcal{S}_{g \downarrow} \cap \mathcal{S}_{g \uparrow}=\mathcal{S}_{T}=\mathcal{S}_{[]}$for any $\mathcal{S}$ closed under primitive recursive operations. It is conjectured in [5] that $\mathcal{S}_{g \downarrow} \neq \mathcal{S}_{g \uparrow}$. Theorem 12 shows that this conjecture holds. The following theorem will be used as a lemma in the proof of Theorem 12. Its proof can be found in Georgiev et al. [2]. 
Theorem 11. For any subrecursive class $\mathcal{S}$ that is closed under primitive recursive operations, we have

$$
\text { (i) } \mathcal{S}_{T \uparrow} \cap \mathcal{S}_{D}=\mathcal{S}_{g \uparrow} \quad \text { and } \quad \text { (ii) } \mathcal{S}_{T \downarrow} \cap \mathcal{S}_{D}=\mathcal{S}_{g \downarrow} .
$$

Theorem 12. For any subrecursive class $\mathcal{S}$ that is closed under primitive recursive operations, there exist irrational numbers $\alpha$ and $\beta$ such that

$$
\text { (i) } \alpha \in \mathcal{S}_{g \downarrow} \backslash \mathcal{S}_{g \uparrow} \quad \text { and } \quad(\text { ii }) \beta \in \mathcal{S}_{g \uparrow} \backslash \mathcal{S}_{g \downarrow} .
$$

Proof. Pick an honest function $f$ such that $f \notin \mathcal{S}$. We have $\alpha^{f} \in \mathcal{S}_{T \downarrow}$ by Theorem 6 , and we have $\alpha^{f} \in \mathcal{S}_{D}$ by Corollary 8. By Theorem 11, we have $\alpha^{f} \in \mathcal{S}_{g \downarrow}$. By Theorem 5, we have $\alpha^{f} \notin \mathcal{S}_{g \uparrow}$. This proves (i). The proof of (ii) is symmetric.

\section{References}

1. Georgiev, I.: Continued fractions of primitive recursive real numbers. Mathematical Logic Quarterly 61 (2015), 288-306.

2. Georgiev, I., Kristiansen, L. and Stephan, F.: Subrecursive Approximations of Irrational Numbers by Variable Base Sums.

ArXiv e-prints (2018), arXiv:1804.05330 [math.LO].

3. Ko, K.: On the definitions of some complexity classes of real numbers. Mathematical Systems Theory 16 (1983), 95-109.

4. Ko, K.: On the continued fraction representation of computable real numbers. Theoretical Computer Science 47 (1986), 299-313.

5. Kristiansen, L.: On subrecursive representability of irrational numbers. Computability 6 (2017), 249-276.

6. Kristiansen, L.: On subrecursive representability of irrational numbers, part II. Accepted for publication in Computability (a preprint is available from the journal's homepage).

7. Kristiansen, L., Schlage-Puchta, J.-C. and Weiermann, A.: Streamlined subrecursive degree theory. Annals of Pure and Applied Logic 163 (2012), 698-716.

8. Mostowski, A.: On computable sequences. Fundamenta Mathematica 44 (1957), 37-51.

9. Labhalla, S. and Lombardi, H.: Real numbers, continued fractions and complexity classes. Annals of Pure and Applied Logic 50 (1990), 1-28.

10. Lehman, R. S.: On Primitive Recursive Real Numbers. Fundamenta Mathematica 49, Issue 2 (1961), 105-118.

11. Péter, R.: Rekursive funktionen. Verlag der Ungarischen Akademie der Wissenschaften, Budapest, 1957. [English translation: Academic Press, New York, 1967]

12. Rose, H. E.: Subrecursion. Functions and hierarchies. Clarendon Press, Oxford, 1984.

13. Specker, E.: Nicht konstruktiv beweisbare Satze der Analysis. Journal of Symbolic Logic 14 (1949), 145-158. 